\title{
El museo nacional: pasado, presente y futuro
}

\author{
José Óscar Batres Posada \\ Arquitecto \\ Especialista en museografía \\ obatres@cultura.gob.sv
}

\section{Resumen}

El presente documento es una recopilación de datos que permiten reconstruir algunos de los hechos de la vida cultural del Museo Nacional "Dr. David J. Guzmán", donde no menos importante es toda su labor desarrollada hasta este momento, que en los albores de celebrar sus 130 años de fundación. También es meritorio destacar el aporte de su personal técnico así como de gente altruista al devenir histórico del museo, que sin duda ha sido un trabajo lleno de satisfacción por el servicio brindado en favor del conocimiento del público visitante.

El Museo Nacional de Antropología se constituye como el primero en nuestro país y ha sido hasta estos días el principal referente de las investigaciones que hoy permiten enlazar los eslabones de nuestra historia cultural. Construir sus antecedentes como institución no es tarea fácil, ya que muchos detalles por algún motivo no se registraron en su momento. Estas líneas serán solamente una aproximación descriptiva, que se recopila con la información disponible y la experiencia vivenciada.

Palabras clave: Museo Nacional de Antropología, museología, museografía, conservación, difusión cultural.

\begin{abstract}
This document is a compilation of information which will allow for the reconstruction of some of the incidents in the cultural life of the "Dr. David J. Guzmán" National Museum. The development work done up until this moment is no less important given that the Museum is about to celebrate the 130th anniversary of its foundation. It is worthwhile to stress the contributions of the museum's technical personnel in the same way as the contributions of donors in the historical transformation of the National Museum. Without a doubt, work
\end{abstract}


for the museum has been very satisfying, dedicated to the service of providing knowledge to the visiting public.

The National Museum of Anthropology has become the premier museum in our country and, in recent years, it has been the principal guide for conducting research to create meaningful connections in our cultural history. Reconstructing its history isn't an easy task given that many of the details, for various reasons, were not recorded in the moment they occurred, This document will be a an approximation of what happened compiled through all the information that is currently available and through lived experience.

Key words: National Museum of Anthropology, museum studies, museography, conservation, cultural diffusion

\section{Nacimiento del museo}

Durante el siglo XIX, la creación masiva de museos en diferentes países del mundo llevará implícita una teorización acerca de las cuestiones museológicas, las que serán desarrolladas no solo por los profesionales que trabajan en museos, sino también por personalidades de diferentes ámbitos de la cultura.

En América, a diferencia de Europa, los museos sirvieron para el afianzamiento de los sentimientos históricos y nacionalistas. En 1823 se crearon los museos nacionales de Bogotá y Buenos Aires, y en 1825 ocurrió lo propio con el museo nacional de México donde, según Lacouture (1994), "nacen de esta manera los museos de identidad nacional con un discurso histórico para fomentar el arraigo de lo propio y el sentimiento nacional".

Para finales del siglo XIX, el movimiento museístico europeo estaba consolidado; el interés de los museos estaba centrado en la expansión de sus colecciones a partir de los más diversos objetos procedentes de diferentes culturas.

Es muy probable que nuestro país se viera fuertemente influenciado por el ambiente cultural imperante, impulsado por las corrientes europeas. Otro hecho importante relacionado con la museística tiene lugar en 1882, cuando a un lado del Museo de Louvre, en París, se creó la primera escuela dedicada a la formación profesional en asuntos de conservación y restauración de objetos de arte, L'Ecole du Louvre (Linares, 2008).

\section{Marco legal, decreto de creación}

“EL PODER EJECUTIVO DE LA REPÚBLICA DE EL SALVADOR, considerando: Que para facilitar el cumplimiento del Decreto Legislativo del $1^{\circ}$. de febrero del corriente año (1883), es conveniente crear un Museo de los productos naturales e industriales del país; que tal instituto está 
llamado a fomentar los intereses económicos e intelectuales de la República; siendo además reclamado por el estado de cultura del pueblo, ha tenido a bien decretar y DECRETA: Artículo $1^{\circ}$.- Se Establecerá en la capital de la República un Museo de productos minerales, botánicos, zoológicos y manufacturados, con una sección de antigüedades, historia y bellas artes."

\section{Primera dirección del museo nacional}

La tarea de dirigir esta institución cultural por primera vez recae en la persona de David Joaquín Guzmán. Nació en la ciudad de San Miguel el 15 de agosto de 1845, hijo de don Eufrasio Guzmán, expresidente de la República (1844-45 - 1958-59) y de doña Ana María Martorell de Guzmán. Realizó sus primeros estudios en el prestigiado Colegio de Jesuitas de Guatemala y continúo sus estudios de secundaria en la Universidad de San Carlos, obteniendo el grado de Bachiller en Filosofía.

En 1863 viajó a París, donde siguió la carrera de Medicina y Cirugía, doctorándose finalmente en 1867. Ejerció su carrera como profesional en Madrid, España brevemente y posteriormente regresó al país para dedicarse a su especialidad.

Desarrolló una amplia trayectoria casi ininterrumpida de cargos públicos entre 1871 y 1914, entre los relacionados con las actividades culturales destacan los nombramientos siguientes:

En 1872 fue nombrado director de la Biblioteca Nacional.

En 1883, por iniciativa del Dr. Guzmán y bajo los auspicios del gobierno del Dr. Rafael Zaldívar, fue fundado el 9 de octubre el museo nacional, del cual fue su primer director.

En 1887 nuevamente es nombrado director del museo nacional y organizador del jardín Botánico.

En 1898 el Dr. Guzmán es llamado por el gobierno de Nicaragua para fundar en Managua el museo nacional, del cual fue organizador y primer director.

En 1902 el Dr. Guzmán es llamado por el gobierno del Gral. Tomas Regalado, nombrándolo director del museo nacional, y de la Exposición Permanente de Industrias Extranjeras, director de la revista Anales del museo nacional y Jardín Botánico.

En su desempeño como funcionario público, el Dr. David J. Guzmán tuvo la oportunidad de representar en múltiples ocasiones al país como comisionado general de Exposiciones Internacionales. ${ }^{1}$

1 Datos biográficos concretos referentes al Dr. David Joaquín Guzmán, 1845-1945. 


\section{Los inicios del museo}

Las menciones iniciales sobre la creación del museo nacional son relativas y a su vez escasas a las fuentes informativas disponibles. Sin embargo, para tratar de construir una parte de su historia, casi siempre se tiene de referente principal al Dr. David Joaquín Guzmán, su primer director, quien en su estadía en Europa durante su formación profesional adquirió una nueva visión propia de la ilustración europea característica de finales del siglo XIX.

Es importante decir que durante este período Francia se encontraba atravesando significativos procesos de cambio social, político y cultural, estas transformaciones se verán reflejadas en diferentes ámbitos de la cultura francesa, que está estrechamente relacionada con el desarrollo cultural del mundo occidental, en particular en las áreas de las artes y de las letras, pues París para esa época era considerada como el punto origen de la cultura europea moderna. ${ }^{2}$

El entorno cultural en el que se desarrolló el Dr. Guzmán pudo ser el germen inspirador para proponer, ante las autoridades estatales del momento, contar con un museo en nuestro país, que se consolidó el 9 de octubre de 1883, coincidiendo con lo que acontecía en Europa debido al surgimiento de muchos museos, promovidos por los cambios sociales que se impulsaron a través de las diversas expresiones artísticas y apertura de muchos espacios museísticos (Batres, 2013).

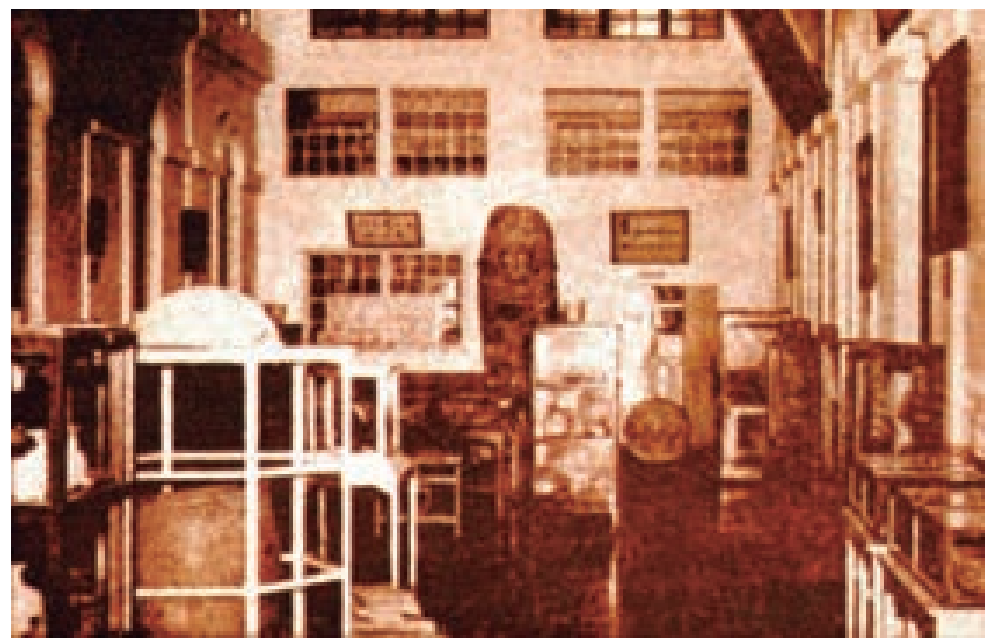

Imagen 1. Aspecto que ofrecía la exhibición permanente del museo nacional cuando se encontraba instalado la Finca Modelo a un costado de la ex Casa Presidencial. Fotografía cedida por Sandra Alarcón.

2 Consultado en: https://publicaronline.wordpress.com/2008/10/15/francia-educacion-culturareligion-lenguas-musica-biblioteca-y-museos/ 


\section{Sedes del museo nacional}

La antigua Universidad Nacional, la casa "Villa España" (antigua residencia de la familia Sagrera, ubicada en la calle Arce y 7.a avenida Norte) y pabellones en la finca Modelo, fueron en su momento algunas de las sedes que ocupó el museo nacional.

La historia de algunos museos que surgieron en aquella época probablemente compartió similares experiencias. Muchos museos han surgido por la iniciativa visionaria de un interesado en preservar el patrimonio cultural, necesidad que casi siempre estuvo dentro de las instalaciones de más de una universidad. No es caso generalizado, pero tampoco es extraño, que hayan surgido producto de la acumulación de colecciones encontradas por casualidad, o de colectas de objetos agrupados por curiosidad o en casos específicos pretendiendo rescatar objetos de interés cultural que desde sus hallazgos fueron destinadas a la investigación científica y que con el pasar del tiempo crecieron en número, dando origen a un importante acervo patrimonial. La actividad museológica en nuestro país, proyectada en perspectiva incipiente, pudo haber sido impulsada más por la curiosidad que por métodos científicos.

Pocas son las referencias acerca de los antecedentes del museo nacional. $\mathrm{Su}$ historia se comienza a construir desde el primer espacio que ocupó, mencionándose que fue dentro del antiguo edificio de la Universidad Nacional, posteriormente se ubicó en diferentes espacios, entre ellos los siguientes:

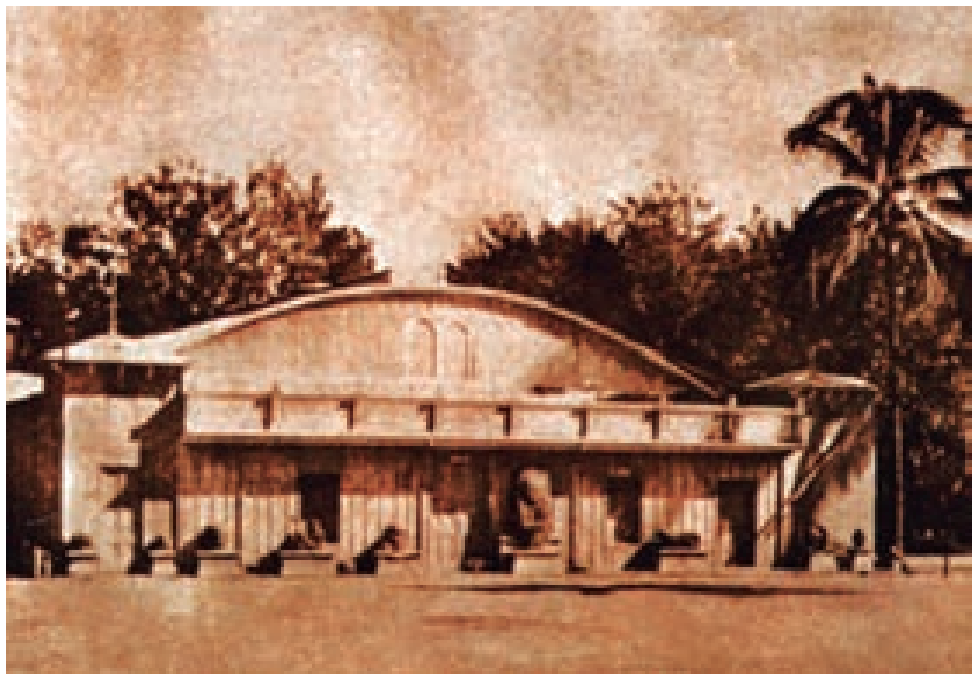

Imagen 2. La estela de Tazumal y algunos petrograbados adornaban la fachada de la Finca Modelo a principios de siglo XX. Fotografía cortesía de Sandra Alarcón. 
- Casa "Villa España", en 1902.

- Finca Modelo en 1904.

- En 1911, la Secretaría de Fomento cierra el museo nacional.

- En 1912 se abre nuevamente el museo, anexado a la Facultad de Química y Farmacia de la Universidad Nacional.

- En 1913 se ubica nuevamente en la Finca Modelo junto al Instituto de Historia Natural y al Jardín Botánico.

- Desde 1927 ocupó los pabellones de Casa Presidencial hasta 1962.

- En 1945 el Ministerio de Cultura Popular, en solemne acto oficial, denominó el museo nacional con el nombre del Dr. David J. Guzmán, con motivo del centenario del nacimiento de su fundador y primer director.

- El 9 de octubre de 1962 se trasladó al edificio construido para el museo nacional, ubicado sobre la avenida La Revolución, en la colonia San Benito; último que utilizó hasta 1994 y que fue demolido por fallas estructurales del terremoto del 9 de octubre de 1986, dando paso al nuevo edificio diseñado y construido en la misma zona y el mismo terreno, el cual fue ampliado debido a las nuevas necesidades que demandaban los requerimientos del diseño para su funcionamiento, contando con un área de terreno mucho mayor a la que se disponía (Batres, 2003).

\section{Primer espacio permanente del museo nacional: conceptualización, zonificación espacial, diseño museográfico}

Considerando que las primeras colecciones que albergaba el museo estaban comprendidas en objetos, botánicos, zoológicos, manufacturados y de mineralogía, con una sección de antigüedades, historia y bellas artes, su orden no sufrió cambios sustanciales en la presentación de sus variados acervos comprendidos por colecciones de interés cultural y natural, concepto museológico y museográfico que se mantuvo hasta finales de la década de los sesenta.

También se presentaba de manera preocupante la situación de sus espacios, pues desde su fundación el museo nacional ocupó muchos locales para desarrollar sus actividades. Y fue el presidente José María Lemus (14 de septiembre de 1956 26 de octubre de 1960), quien instruyó que se diseñara y construyera un edificio para el museo nacional, ya que desde 1927 había ocupado los pabellones de la antigua Casa Presidencial hasta 1962, año en que se realiza su trasladado a las nuevas instalaciones ubicadas sobre la avenida La Revolución de la colonia San Benito, en San Salvador, para ocupar y desarrollar su labor difusora de la cultura nacional.

Probablemente, al momento de su apertura en su nueva ubicación, mantuvo el mismo concepto de exhibición que caracterizaba a los museos polivalentes, mostrando las colecciones de diversa índole, incorporadas con colecciones prehispánicas, históricas y etnográficas. 


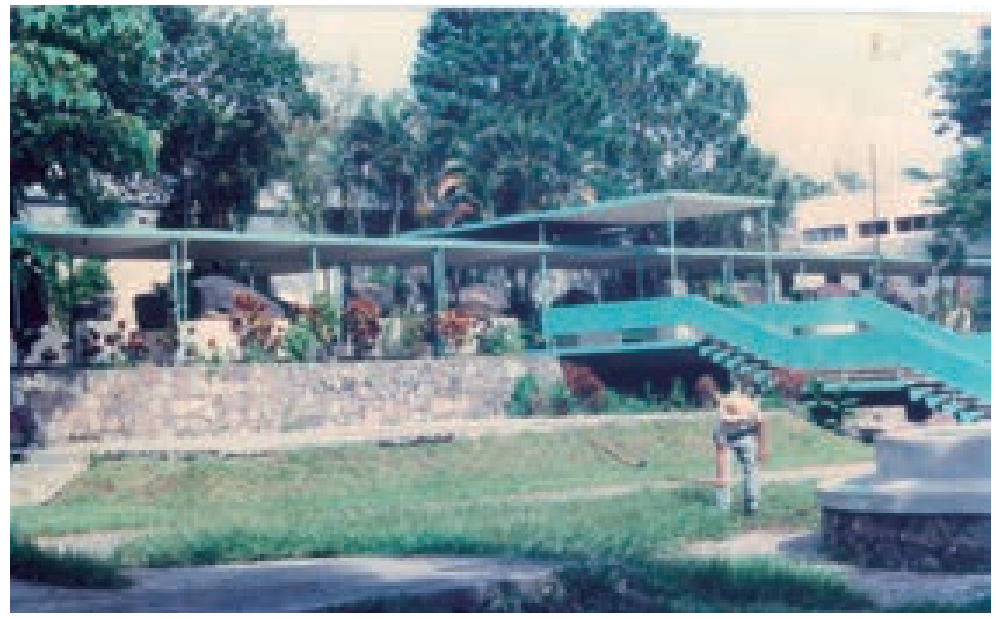

Imagen 3. Fachada principal del antiguo museo nacional ubicado en la avenida La Revolución de la colonia San Benito, en la que se observa el pasillo rupestre. Fotografía cortesía de Sandra Alarcón.

Este nuevo edificio también soportó las consecuencias del terremoto del 3 de mayo 1965; y durante el tiempo que estuvo habilitado para los servicios públicos también se sucedieron aspectos importantes en el devenir de su funcionamiento, pues a partir de principios de la década de los setenta se propician cambios institucionales, y en 1974 se crea la Administración del Patrimonio Cultural; se construye a su vez el edifico administrativo para el funcionamiento de su estructura técnica y administrativa, donde se ubicaban las siguientes unidades laborales: Departamento de Museos, Departamento de Investigaciones, Departamento de Sitios y Monumentos, además de un área administrativa, constituyéndose así en la columna vertebral de lo que a futuro sería el desarrollo de esta instancia estatal.

El museo nacional se mantuvo como la unidad rectora y competente para la administración y manejo de los dos museos que dependían de la Dirección de Patrimonio Cultural, el museo nacional y el museo arqueológico del sitio Tazumal, con la nueva estructura también se replantearon los contenidos informativos de las salas de exhibición, y desde los inicios de la década de los setenta se establece la delimitación del manejo de colecciones, enfocando su interés a los contenidos de carácter prehispánico, histórico y etnográfico.

Otro evento natural azota las instalaciones del museo el 10 de octubre 1986; un fuerte terremoto afecta la ciudad de San Salvador. El sismo daña seriamente la estructura de soporte de las salas de exhibición. Luego de este acontecimiento se procedió a cerrar sus instalaciones en 1993 para evitar accidentes en los visitantes y empleados del museo y daños en la colección. Las oficinas administrativas y las áreas técnicas fueron las únicas instalaciones que estuvieron habilitadas, hasta mediados del año 1996, para dar inicio a la demolición del edificio. 


\section{Formación de la colección del museo}

La creación de las primeras colecciones de bienes culturales muebles de El Salvador se ubica en el contexto de los años 1870 a 1890 , época en que el principal referente cultural, artístico y científico para un gran segmento de la clase alta y media de nuestro país era Francia.

Es así que en este ambiente surge la figura del Dr. David J. Guzmán que, cuya influencia e impacto que crean en él el esplendor y la "magnificencia" de los museos franceses, al retornar a nuestro país, con el apoyo de algunos intelectuales, artistas y amigos como son Juan J. Cañas, Juan Aberle y otros, hace que proponga al gobernante de la época, Dr. Rafael Zaldívar, la creación del primer museo estatal en El Salvador. Es así como, el 9 de octubre de 1883, se publicó el acuerdo de creación del museo nacional.

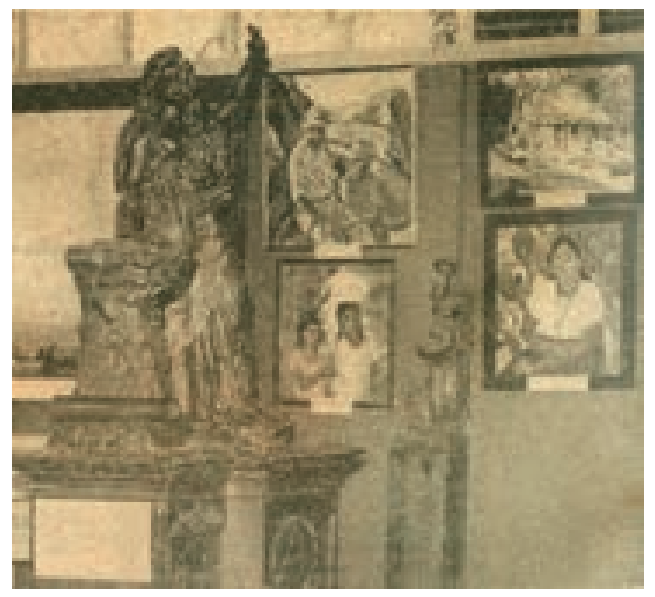

Imagen 4. Muestra de las colecciones que albergaba el museo nacional y que posteriormente se trasladaron durante la especialización temática a otros espacios de exhibición a finales de los años 60. Fotografía cortesía de Sandra Alarcón.

Considerando el carácter de colecciones con las que inició el museo, el cual mostraba una diversidad, revela la clase y naturaleza de las primeras colecciones o muestras que exhibía, siendo su principal fuente o forma de adquisición las donaciones, préstamos y posiblemente algunas compras de objetos.

Esta misma tónica de adquisición de colecciones se ha mantenido a través de los años hasta nuestros días con ligeras variantes, siendo la más predominante la adquisición por donaciones. Esto lo confirma la actual base de datos de las colecciones del museo, que indica que aproximadamente entre el 70 y el $75 \%$ de las colecciones actuales no tienen contexto, son de origen desconocido.

Con la separación de las colecciones durante la década de los setenta, se procedió a la creación del Museo de Historia Natural, por lo que las colecciones concernientes a esta temática fueron trasladadas a dicho museo. Debido al carácter antropológico que asumía el museo nacional "David J. Guzmán", 
solamente quedan en sus recintos las colecciones nacionales de Arqueología, Historia y Etnografía, siendo la primera la más predominante.

Algunos de los primeros investigadores, arqueólogos empíricos o aficionados que a través de su recolección de los primeros objetos o materiales arqueológicos sirvieron para construir las colecciones nacionales fueron Santiago I. Barberena, Atilio Peccorini, Jorge Lardé, Fidias Jiménez, entre otros.

Algunos de los objetos o piezas emblemáticas de la colección arqueológica que subsisten en la actualidad y que son de las primeras colecciones del museo son: la Estela de Tazumal, el Chacmool, el disco Solar de Cara Sucia, el Jaguar esculpido en piedra. Así mismo, entre los objetos de la colección de historia que más se destacan $-\mathrm{y}$ posiblemente fue de los primeros objetos que se adquirieron para el museo - están los siguientes: la colección de armas y objetos coloniales, la colección de objetos relacionados con Gerardo Barrios, algunas obras escultóricas de Pascasio Gonzáles ("Minerva" y "Mariscal Santiago Gonzáles"), el bastón de don Juan Aberle, el tintero de Juan J. Cañas, el tintero del Dr. David J. Guzmán, y el busto del Dr. Rafael Zaldívar (expresidente de El Salvador) y un sable de su pertenencia.. En la actualidad, las principales fuentes o formas de obtención de piezas para la colección arqueológica nacional son por investigación, donación y decomiso.

La colección etnográfica nacional ha sido producto principalmente de compras y de algunas donaciones, como resultado de renovaciones que se han hecho a través de los años en las salas del museo, siempre que ha surgido la necesidad de sustituir o reemplazar algún material deteriorado o perecedero, o cuando se ha planteado la necesidad de enriquecer la temática o naturaleza del guion museográfico diseñado (Rubio, 2013).

\section{Descripción de las salas de exhibición}

A partir de la creación de la Administración del Patrimonio Cultural, se consolidan algunos cambios al interior del museo. Se determinó como prioridad que el desarrollo de las investigaciones estuviera enfocado a lo antropológico e histórico para dar una base firme al carácter del museo, definido así por el tipo de colecciones que venía mostrando desde los inicios de su creación. Con la estructura funcional y enfoque científico que ya reflejaba, la Administración especificó su contenido temático para mostrar con mayor énfasis su enfoque en los aspectos arqueológicos, históricos y etnográficos.

En consonancia con los cambios dados a principios de la década de los setenta, dentro de la estructura del Ministerio de Educación y su nuevo enfoque dirigido a los sistemas de instrucción formal, se mantenía la idea primordial que perseguía el museo de contribuir mediante la educación no formal, enfatizando la necesidad del rescate y difusión de los aspectos sobresalientes de la cultura 
salvadoreña, por lo que el nuevo enfoque del museo era presentar una secuencia evolutiva de la presencia humana en nuestro territorio desde momentos muy tempranos, enfocando un desarrollo histórico que abarcaba desde los inicios de la época prehispánica del actual territorio de El Salvador hasta nuestros días.

La distribución y organización de las áreas de exhibición estaba sugerido en un orden cronológico, manteniendo una secuencia ininterrumpida en todo el recorrido del museo y dispuesta de la siguiente manera:

\section{a) Sala Introductoria}

Esta se constituía en el área de ingreso al museo, ubicada en el área vestibular, y sus elementos de exhibición eran la maqueta del relieve topográfico de la República de El Salvador, que ubicaba algunos lugares culturales importantes, entre ellos los sitios arqueológicos, algunas iglesias y vestigios coloniales en nuestro país.

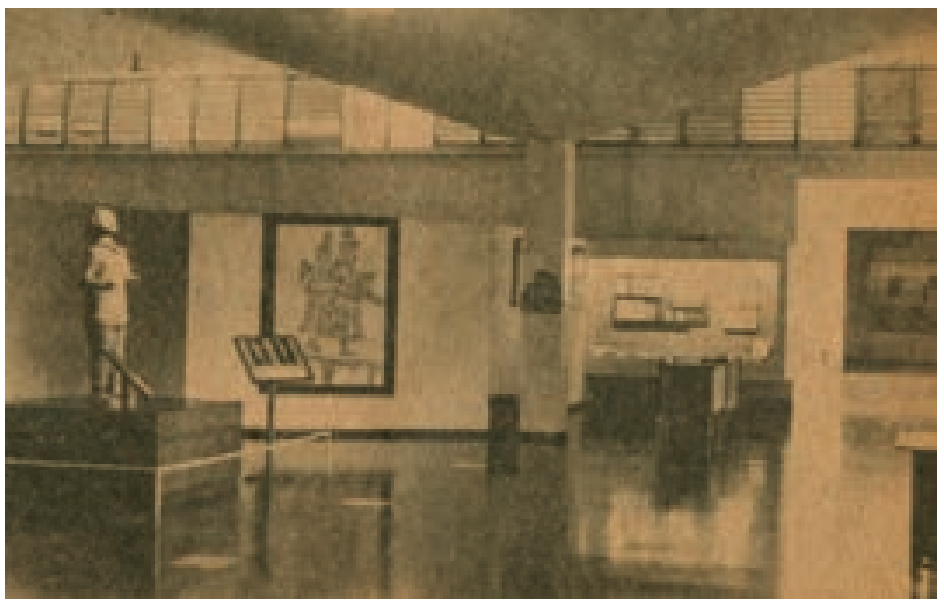

Imagen 6. Sala introductoria del antiguo museo nacional en la avenida La Revolución. Fotografía cortesía de Sandra Alarcón.

\section{b) Sala de Arqueología}

Iniciaba su introducción con el planteamiento de la formación del puente natural de hielo en el estrecho de Bering, por donde se supone se produjo parte del fenómeno de las migraciones para poblar lo que hoy es América. Se agregaban a esta información los fragmentos óseos de mastodonte para relacionar el contexto geográfico en esta región del continente.

En la exhibición se presentaban las áreas geográficas de los sitios prehistóricos del Valle de México y finalizaba con la muestra de materiales más antiguos que reflejaban presencia humana en El Salvador, entre ellos la Gruta de Corinto, 
localizada en el departamento de Morazán; su colección estaba conformada por puntas, lascas, raspadores y perforadores.

\section{c) La Sala Conceptual}

Presentaba esquemáticamente el área geográfica y cultural conocida como Mesoamérica. Su objetivo era ubicar al visitante sobre las principales zonas de asentamiento de los grupos culturales que ejercieron su desarrollo e influencia en cada uno de los momentos comprendidos a partir de los períodos preclásico (1500 a.C.-250 d.C.), clásico (250-950 d.C.) y postclásico (950-1525 d.C.).

Se presentaba también una maqueta estratigráfica con la idea de visualizar las deposiciones culturales a lo largo de los tres períodos de la época prehispánica en El Salvador. Esto era con el fin de relacionar momento de ocupación con el contenido de las salas, asociando el fechado con los artefactos culturales encontrados, siendo estos los diversos objetos distribuidos en las salas de exhibición.

\section{d) La Sala Preclásica}

Pretendía mostrar las evidencias arqueológicas producidas a lo largo de un período aproximado de 750 años de actividad de los grupos pobladores diseminados en distintas zonas geográficas del país. Entre los objetos que se exhibían se destacaba la muestra de cerámica utilitaria que procede del área de Chalchuapa, las figurillas Bolinas, la cerámica Batik Usuluteco, así como la muestra de sellos prehispánicos con motivos geométricos y relieves zoomorfos (formas de animales) y relieves fitomorfos (formas de plantas). En este período se describía la existencia de contactos con el grupo cultural olmeca y su presencia evidenciada dentro del área de Chalchuapa.

\section{e) La Sala Clásica}

Desarrollaba una breve referencia de la cultura durante el período clásico a través de la muestra de variados objetos y su diversidad de

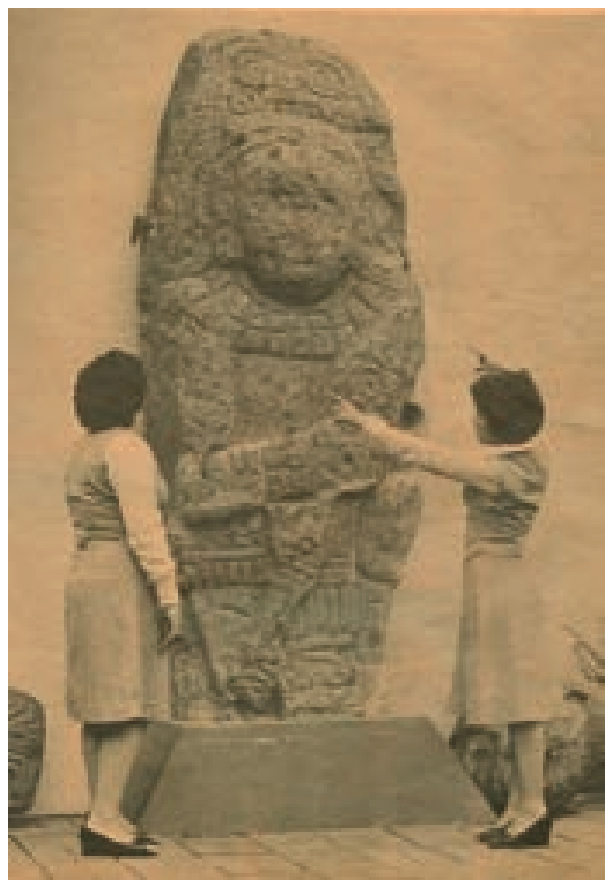

Imagen 7. Dos guías del museo nacional observan la estela de Tazumal en las instalaciones de la primera sede del museo en la colonia San Benito. Fotografía cortesía de Sandra Alarcón. formas y diseños policromos que 
fueron elaborados en un período aproximado de 750 años, en el que se hacía sentir probablemente la influencia de grupos humanos asociados con la cultura maya. Algunos de los objetos provenientes de los distintos sitios arqueológicos eran vasos, cajetes, figurillas y objetos tallados en jade, entre otros. Los sitios mencionados eran Tazumal, Cara Sucia, Asanyamba, Quelepa y otros. En la muestra cerámica de este período destacaban las colecciones de cerámica Salúa y Copador por sus variados motivos y colores utilizados.

\section{f) La Sala Postclásica}

Presentaba un breve panorama sobre las evidencias materiales del hombre de este período, donde es muy sobresaliente la cerámica Plomiza, los objetos provenientes del sitio arqueológico Cihuatán y los provenientes de los diversos sitios del área de San Lorenzo, que correspondían al rescate arqueológico realizado por la Dirección de Patrimonio Cultural, comprendido por objetos cerámicos del estilo policromo Nicoya y Anaranjado Fino.

\section{g) Sala de Historia}

Esta sala mostraba un panorama general de los acontecimientos más importantes que se relacionan con la presencia española en tiempos de la Conquista y colonización en El Salvador. Los objetos mostrados se describían momento a momento desde la incursión de don Pedro de Alvarado en la Conquista de Cuscatlán hasta el período de la independencia patria alcanzada en 1821, concluyendo con la muestra de objetos que se referían al desarrollo tecnológico en nuestro medio impulsado por los aportes de la Revolución industrial.

\section{h) Sala Etnográfica}

Su intención era brindar una visión, si se quiere, folclorista de la cultura tradicional de El Salvador; mostraba una idea generalizada de los aspectos costumbristas y tradicionales que se manifiestan a través de ciertos fenómenos y patrones de conducta asociados a la cultura popular, algunas de las cuales todavía se ponen de manifiesto en ciertas poblaciones con el hombre actual. Los objetos que conformaban la colección etnográfica eran mostrados en su conjunto y procedían de centros artesanales muy reconocidos como Nahuizalco, Guatajiagua, Ilobasco, Quezaltepeque, entre otros. La Sala se resumía en tres módulos de exhibición que mostraban la religiosidad popular, la vivienda tradicional y se complementaba con el comercio tradicional que mostraba una diversidad de objetos cerámicos, artesanías en fibras duras, textiles, etc.

\section{i) Sala de Exposiciones Temporales}

Debido a la necesidad de atender otros servicios de difusión, se acondicionó un segmento del museo destinado a muestras de diversas temática. La existencia de esta sala dentro del recinto del museo nacional era con el fin de dar cabida 
al desarrollo de exhibiciones con cierto período de duración, se instalaron exhibiciones con colecciones nacionales desarrollando temas específicos sobre Arqueología, Historia y Etnografía; así también hubo muestras de exposiciones internacionales y de colecciones privadas (Batres, 2003).

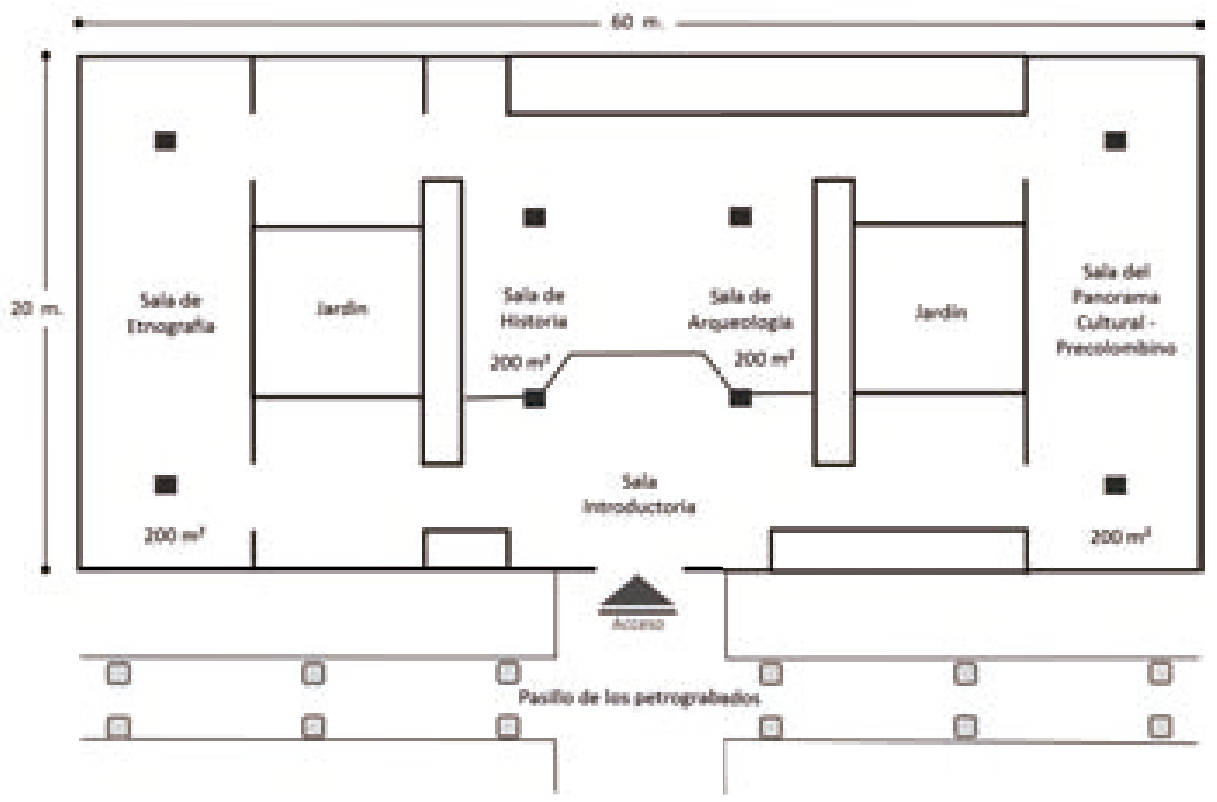

Figura 1. Reconstrucción espacial del antiguo museo nacional en la colonia San Benito. Fuente: Arq. Óscar Batres, 2015

\section{Consolidación del Museo Nacional}

Debido al terremoto de 1986, el antiguo edificio del museo nacional quedó gravemente afectado en su estructura física, lo que aceleró la decisión de las autoridades estatales para construir un nuevo edificio sede.

Este aspecto puede atribuirse compromisos institucionales que asumió en su momento el presidente de Concultura de turno. En este caso correspondió ${ }^{3}$ a don Roberto Galicia, que con una visión sensible a la proyección de la cultura nacional, tuvo a bien presentar como principal proyecto de su gestión el diseño y construcción del nuevo edificio del Museo Nacional de Antropología presentado al entonces presidente Dr. Armando Calderón Sol. Galicia, conocedor de la realidad nacional en términos de la administración cultural, buscó dejar para el país a través del museo nacional un espacio permanente que sirviera no solo para conocer, sino también para investigar y dignificar la cultura nacional.

3 1994-1999 período presidencial del Dr. Armando Calderón Sol 
La idea de establecer un nuevo edificio para el museo nacional tuvo sus específicas motivaciones, entre ellas, hacer sentir implícitamente que se merecía hacer un reconocimiento al trabajo científico iniciado por Stanley Boggs y Concepción Clará de Guevara, entre otros investigadores. Esto como una continuidad del trabajo iniciado de manera formal en la década de los setenta, de la que también el señor Galicia de alguna forma fue artífice de estos primeros cambios en el devenir de la cultura nacional.

Se diseñó una completa estrategia de trabajo dirigida por la arquitecta María Isaura Aráuz, Directora Nacional de Patrimonio Cultural. El primer paso fue establecer el proceso de formulación del guion temático y las investigaciones correspondientes, labor que fue realizada por el personal de investigadores de la Dirección Nacional de Patrimonio Cultural; las investigaciones arqueológicas estuvieron coordinadas durante este período por el Lic. Vicente Genovés, y las investigaciones antropológicas fueron encausados por el Lic. Carlos Benjamín Lara.

\section{El nuevo edificio: la sede definitiva del museo nacional con un diferente concepto de diseño arquitectónico y de contenido}

En 1996, fue posible diseñar y construir un edificio digno para exhibir lo más representativo de la cultura nacional. Roberto Galicia propuso al presidente de la República el proyecto de diseño y construcción del nuevo edificio, dando inicio a los preparativos con el desarrollo de un concurso de ideas para el diseño de la nueva sede, al que atendieron dieciséis empresas nacionales vinculadas con el diseño y construcción arquitectónicos. Con la idea básica se perfilaría el funcionamiento espacial para el edificio que debería de contener una infraestructura adecuada y capaz de brindar nuevos y mejores servicios al público, además de propiciar en su funcionamiento una visita mucho más interesante dentro de las salas de exhibición. Este fue uno de los primeros edificios planificados para un museo, que reflejaría espacialmente el orden para el desarrollo de sus diferentes funciones técnicas y administrativas.

\section{Su conceptualización}

Para la ejecución de este proyecto es necesario hacer notar la importancia que se le imprimió desde la presidencia de Concultura a su desarrollo, depositando esta responsabilidad en la Dirección Nacional de Patrimonio Cultural, sobrellevando la responsabilidad de definir dos aspectos importantes: el diseño del edificio y el guion del contenido temático y museográfico.

Se formuló una solicitud de apoyo a otras instituciones especializadas con este tipo de experiencias. Esta se obtuvo mediante una consultoría técnica de carácter internacional para contar con la presencia del arquitecto y museólogo Felipe Lacouture Fornelli, enviado especial del Instituto Nacional de Antropología e 
Historia de México, cuyas recomendaciones fueron incluidas en el desarrollo del diseño final del edificio.

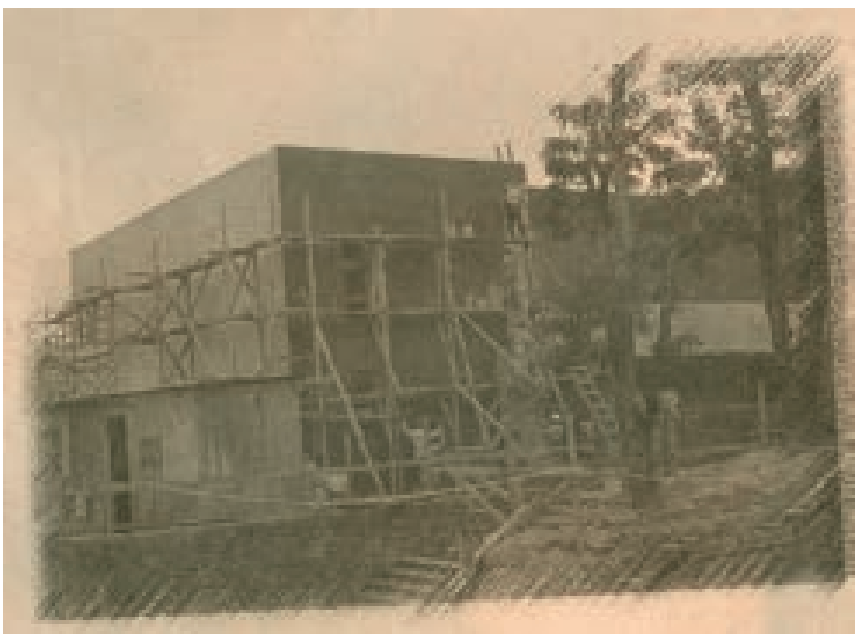

Imagen 8. El actual edificio del Museo Nacional de Antropología en proceso de construcción a finales de los años 90. Fotografía cortesía de Sandra Alarcón.

\section{El edificio}

Desde el año 1994 al 2001, se trabajó en la planificación y organización del nuevo edificio, que se construyó especialmente para el Museo Nacional de Antropología "Dr. David J. Guzmán". El proyecto inició en marzo de 1995 con el lanzamiento del concurso de ideas para el diseño del edificio. Se readecuaron temporalmente los depósitos para las colecciones de bienes culturales muebles; paralelamente se desarrolló la evacuación y reubicación de las oficinas técnicas y administrativas para dar comienzo a las obras de demolición de las antiguas instalaciones del museo.

El diseño del edificio corresponde a los arquitectos salvadoreños Roberto Dada y Francisco Altschul, quienes ganaron el concurso de ideas del diseño arquitectónico; y las obras de construcción del edificio estuvieron a cargo del Arq. Rubén Vásquez.

La construcción del edificio comenzó en septiembre de 1997 y finalizó en 1999, manteniéndose una permanente supervisión de la obra a cargo de la Arq. Lilly Lemus de Baños, quien estuvo al frente para garantizar el desarrollo de la obra. Luego de concluidas las obras de construcción, nuevamente se reubicaron las oficinas técnicas especializadas de Museografía, Conservación y Restauración de Bienes Culturales Muebles, Registro e Inventario de Bienes Culturales Muebles, la Biblioteca y Hemeroteca especializada y la unidad de Arqueología dentro de las nuevas instalaciones del museo nacional. 


\section{La zonificación espacial}

La propuesta funcional del edificio respondió a los estándares esperados, considerando que los diseñadores se apegaron al cumplimiento del programa de necesidades para cumplir con lo exigido, así como al documento técnico que contenía los términos de referencia, enfatizando el desarrollo del funcionamiento del edificio en tres grandes zonas, siendo estas las siguientes:

\section{a) Zona pública}

Esta comprendería los espacios de las salas de exhibiciones temporales y permanentes, en espacios abiertos como los patios y el jardín rupestre donde el público de forma ordenada y sorpresiva encontraría información complementaria en su visita. Además el museo presenta una plaza vestibular, el área de información, los espacios del foyer y el balcón en el primero y segundo nivel del edificio, respectivamente; cuenta también con auditórium, área comercial para estancia de los visitantes, servicios de consulta bibliográfica para investigadores dentro de la biblioteca especializada, estacionamiento, entre otros.

\section{b) Zona administrativa}

Se localiza en el segundo nivel del edificio y tiene como función principal velar por el buen funcionamiento y estado de las instalaciones. Así mismo, entre sus funciones está el apoyar y complementar el desarrollo de las actividades técnicas referidas a los servicios dirigidos al público, así como brindar información de carácter administrativo.

\section{c) Zona técnica}

Corresponde al recinto laboral que da vida a las salas de exhibición. Esta zona es el soporte técnico en el que se desarrollan las actividades de control de colecciones, investigación, conservación y preparación de las exhibiciones. Dentro del diseño del edificio se consideró el depósito de colecciones, oficina de registro e inventario de colecciones, museografía, el taller de conservación y restauración; se agregaba dentro del esquema funcional el área de investigaciones y curaduría.

Considerando que en los museos por lo general siempre existe una dinámica interna, en los últimos años en estas instalaciones han existido cambios sustanciales para responder a necesidades diversas, por lo que algunos espacios se han acondicionado para continuar con el desempeño laboral.

\section{El contenido}

El planteamiento conceptual del contenido del museo estuvo a cargo del antropólogo Carlos Lara Martínez, quien con su equipo de colaboradores, investigadores, etnólogos, antropólogos, arqueólogos e historiadores formularon los ejes temáticos para el contenido de cada una de las salas de exhibición. 
Luego de definidos los guiones temáticos y de la selección de las colecciones, se aspiraba a presentar un museo formalmente constituido en el que los investigadores seleccionarían los objetos que estuvieran relacionados con su contexto, se consideró necesario desarrollar la curaduría del contenido, la cual estuvo a cargo del Dr. Ramón Rivas, antropólogo con una amplia trayectoria en el campo de la investigación. Esta parte del historial del museo tenía como propósito consolidar el orden de contenido y secuencia de cada una de las salas de exhibición, que se identificaron de la siguiente manera:

- Sala Conceptual

- Sala de Asentamientos Humanos

- Sala de Agricultura

- Sala de Producción e Intercambio Artesanal

- Sala de Religión

- Sala de Arte y Formas de Comunicación

- Sala Temporal

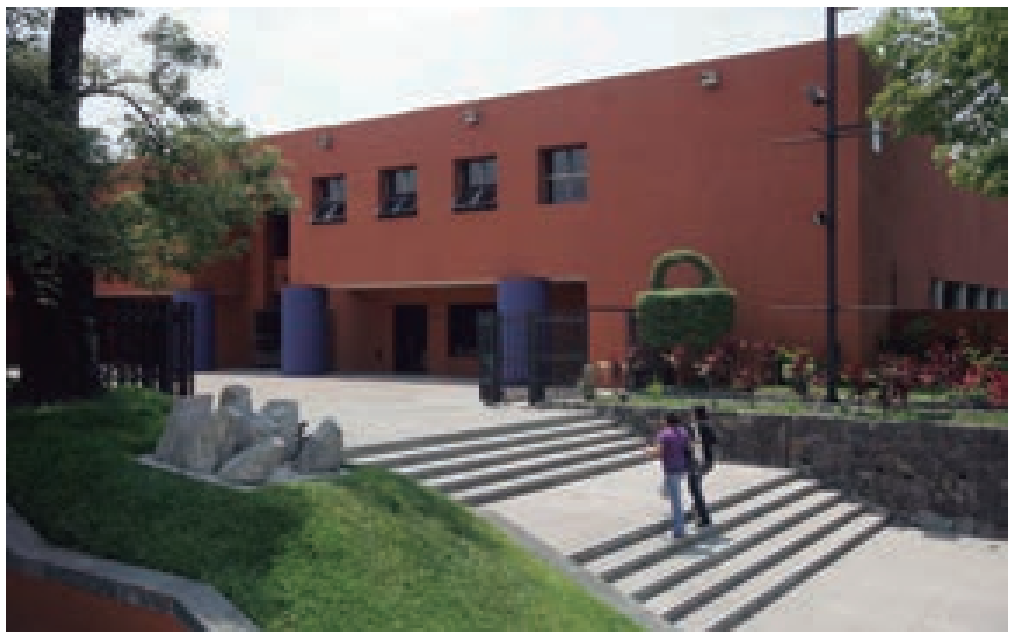

Imagen 9. El actual edificio del Museo Nacional de Antropología. Fotografía cortesía de Sandra Alarcón.

En las salas, en su nuevo planteamiento museológico y museográfico, la presentación de su discurso respondía a principios fundamentales de continuidad y discontinuidad asociados con la dinámica cultural y sus cambios reflejados en tiempo y espacio, tratando de mostrar las actividades del hombre en su entorno.

Además existen otros espacios destinados a exposiciones permanentes y temporales, como son los servicios de atención al público en el área de servicios educativos y el área lúdica. 
Se proyectó una gama de servicios y espacios para convertir al Museo Nacional de Antropología "Dr. David J. Guzmán" en un espacio cultural dinámico, que a su vez se afirmará como el principal exponente de la cultura salvadoreña, que continuara con la noble tarea de mostrar al pueblo, en las presentes y las próximas generaciones, la importancia de no solo conocer y disfrutar, sino también de hacer sentir la necesidad de conservar y difundir el patrimonio cultural salvadoreño.

\section{El diseño y el montaje museográfico}

Entre los años 2000 y 2001 se realizó el proyecto de diseño en las salas de exhibición del nuevo edificio del museo. Estuvo a cargo de una empresa que desarrolló la propuesta del diseño, que estaba bajo la supervisión del Arq. Carlos Candell, quien conformó un equipo técnico de profesionales nacionales y extranjeros para responder a los requerimientos esperados.

\section{El montaje museográfico}

Esta actividad fue ejecutada con un equipo multidisciplinario completo, que conformaba la Dirección Nacional de Patrimonio Cultural, en el que participaron arquitectos, ingenieros, investigadores, historiadores del arte, conservadores, restauradores, museólogos y museógrafos de la entonces Coordinación de Museos Nacionales.

Un hecho anecdótico que no se puede dejar de mencionar es que en el proceso de montaje museográfico previo a su apertura, nuestro país nuevamente es afectado por los terremotos del 13 de enero y del 13 de febrero de 2001. Ambos afectaron significativamente gran parte del patrimonio cultural edificado. En los preparativos de la presentación museográfica, el guion temático de la Sala de Asentamientos Humanos desarrollaba el tema sobre conservación del patrimonio edificado, crecimiento y desarrollo de las ciudades, pero el fenómeno mencionado obligó a modificarlo, replanteando los efectos de la vulnerabilidad y daños irreversibles en algunos casos, que de igual forma afectaron los edificios patrimoniales de los centros históricos. Esto con el fin de dejar un testimonio informativo sobre los daños que afectaron a muchos pueblos y ciudades. La información presentaba gráficamente los daños causados por los sismos en el patrimonio edificado de interés patrimonial para nuestro país.

La nueva museografía fue inaugurada el 9 de octubre de 2001, durante la administración presidencial del Lic. Francisco Flores, fungiendo entonces como presidente de Concultura el Ing. Gustavo Herodier. Fue una museografía proyectada para una duración de no más de diez años (Batres, 2013). 


\section{Renovación de las salas de exhibición}

Luego de casi doce años de que el nuevo museo fuera abierto al público, se tenía claro que la propuesta museográfica debería responder a un período en términos de su concepto museológico y museográfico. Dos aspectos importantes que se consideraron en su momento fueron el deterioro y el vencimiento del material gráfico informativo, así como la actualización de su información, lo que también podría implicar cambios físicos dentro de los espacios de exhibición.

Los primeros cambios internos de carácter museológico y museográfico que se iniciaron en las salas del museo estuvieron bajo la conducción del Lic. Gregorio Bello-Suazo, siendo el primer director en esta nueva fase, cubriendo el cargo durante el período 2004-2009. Tres salas de exhibición fueron sometidas a cambios internos. Una de ellas fue la Sala de Arte y Formas de Comunicación. En este caso se desmontó la colección y fue cerrado su espacio de exhibición de forma completa, solamente se dejó habilitado un segmento para instalar la sala informativa identificada como América Prehispánica, la que mostraba un panorama histórico de algunas de las culturas más importantes del continente americano. Así mismo, cabe mencionar que esta sala sirvió eventualmente para el montaje de algunas exposiciones temáticas temporales, para luego volverla a cerrar.

Otra sala que fue modificada drásticamente en su contenido fue la sala 3, identificada como Sala de Producción Artesanal, Industria e Intercambio, reflejando en su nuevo contenido el predominio de una perspectiva prehispánica en su temática. En esta sala se desmontó parte de la información inicial, fragmentando su contenido para incorporar mayor colección de carácter arqueológico. Luego se modificaron internamente el espacio y el contenido de la sala 4, identificada con el tema de Religión. Este espacio de exhibición fue segmentado para dejar instalada una temática específicamente de orden prehispánico.

Con los cambios que ya mostraban algunas salas de exhibición, sin duda sirvieron para desarrollar temas importantes, sus contenidos tenían una tendencia de contenido inclinado a lo prehispánico; y es meritorio destacar los temas ampliados o retroalimentados basados en un planteamiento que fue el resultado de una investigación. De igual forma, se destaca el montaje de exposiciones temáticas presentadas en la Sala Temporal, con trabajos que fueron dirigidos por el Lic. Gregorio Bello-Suazo y su equipo técnico, que atendió las necesidades museológicas y museográficas del momento (Batres, 2013). 
Directores del Museo Nacional “Dr. David J. Guzmán” Desde 1883 a 2015

\begin{tabular}{|c|c|c|}
\hline $\mathbf{N}^{\circ}$ & Nombre & $\begin{array}{l}\text { Período de nombramiento } \\
\text { y desempeño del cargo }\end{array}$ \\
\hline 1 & Dr. David J. Guzmán & 9 de octubre de $1983-1886$ \\
\hline 2 & Dr. Jorge Aguilar & 15 de enero de 1886 \\
\hline 3 & Dr. David J. Guzmán & 1887 \\
\hline 4 & Dr. Santiago Ignacio Barberena & 20 de noviembre de 1894 \\
\hline 5 & Dr. Nicolás Aguilar & 20 de enero de 1897 \\
\hline 6 & Dr. David J. Guzmán & 1902 \\
\hline 7 & Sr. Carlos Alberto Imery & 25 de febrero de 1927 \\
\hline 8 & Dr. Rafael González Sol & 22 de abril de 1931 \\
\hline 9 & Br. Tomas Fidias Jiménez & 3 de mayo 1940 \\
\hline 10 & Prof. Jorge Hurtarte Monzón & 20 de octubre de 1941 \\
\hline 11 & Arq. Augusto Baratta & 25 de abril de 1942 \\
\hline 12 & Sr. Salvador Sánchez Aguillón & 23 de octubre de 1944 \\
\hline 13 & Br. Tomas Fidias Jiménez & 10 de abril de 1945 \\
\hline 14 & Br. Jorge Lardé y Larín & 8 de febrero de 1949 \\
\hline 15 & Prof. Salvador Cañas & 1 de octubre de 1956 \\
\hline 16 & Br. Tomas Fidias Jiménez & 7 de enero de 1957 \\
\hline 17 & Dr. Manuel Alfonso Fagoaga & 8 de enero de 1968 \\
\hline 18 & Sr. Carlos de Sola & 4 de enero de 1971 \\
\hline 19 & Arq. Pío Salomón Rosales & 26 de enero de 1973 \\
\hline 20 & Srita. Ana Lilian Ramírez & 3 de enero de 1974 \\
\hline 21 & Sr. Roberto Huezo & 7 de noviembre de 1975 \\
\hline 22 & Arq. Julia Alvarenga Jule de Quintanilla & 16 de junio de 1976 \\
\hline 23 & Sr. Armando Quintanilla & 1 de noviembre de 1979 \\
\hline 24 & Mgfo. Roberto Guzmán Aguilar & 1 de septiembre de 1980 \\
\hline 25 & Lic. Gloria Aracely Mejía de Gutiérrez & 1984 \\
\hline 26 & Ing. Saúl Salgado & 1984 \\
\hline 27 & Sra. Marina Zimmer & 1984-1985 \\
\hline 28 & Sr. Manuel Roberto López & 1985-1991 \\
\hline 29 & Mgfo. Jose Óscar Batres Posada & $1991-2000$ \\
\hline 30 & Arq. Margarita Ivette Valle & 2000-2002 \\
\hline 31 & Mgfo. José Óscar Batres Posada & $2002-2003$ \\
\hline 32 & Acéfala & 2003-2004 \\
\hline 33 & Lic. Gregorio Bellosuazo & 2004-2009 \\
\hline 34 & Dr. David Hernández & 2009-2010 \\
\hline 35 & Arq. Lilly Lemus de Baños & 2010-2012 \\
\hline 36 & Arq. Eduardo Ernesto Góchez Fuentes & 29 de mayo de 2012 \\
\hline 37 & Lic. Heriberio Erquicia & 1 de juilo de 2014 a la fecha \\
\hline
\end{tabular}




\section{Reflexión final}

Desde su creación en 1883 hasta la década de los 60 del siglo XX, el Museo Nacional de Antropología tuvo cuatro sedes temporales antes de pasar a tener su actual asentamiento fijo.

Ha sido una institución estatal que ha afrontado el cese de funciones por habérsele considerado en alguna ocasión "improductivo" para la sociedad salvadoreña, pero también su último cierre a finales de los años 90 ha sido para ser renovado y mejorar sus exposiciones tanto en contenido como en espacio para el beneficio del público visitante, que ya no dispone solamente de, poco más, $800 \mathrm{~m}^{2}$, cifra alcanzada por sus antiguas salas de exhibiciones, sino que en la actualidad dispone de casi $12000 \mathrm{~m}^{2}$ de exposiciones permanentes y temporales.

Por otro lado, también ha sido testigo de la evolución del pensamiento académico y del interés del estado por el rescate, conservación y difusión del patrimonio cultural salvadoreño.

Las colecciones iniciales de finales del siglo XIX que contenían botánica, historia natural, mineralogía y etnografía fueron sufriendo cambios con el avance del tiempo y a medida que sus directores supieron orientar las colecciones hacia la antropología y arqueología nacional debido a que muchos de ellos investigaron de manera sistemática estos campos del conocimiento en franco ascenso de interés cultural.

La historia del Museo Nacional de Antropología “Dr. David J. Guzmán” seguirá tejiendo los hilos de la ciencia, que se espera puedan servir para fortalecer los lazos de identidad, que propicie una cultura de apropiación del patrimonio cultural que es de todos los salvadoreños.

\section{Referencias bibliográficas}

Batres Posada, J.O. Los museos estatales en El Salvador administrados por Concultura. Museo nacional de Antropología, San Salvador, 2003.

Batres Posada, J.O. Ubicación de los lugares ocupados por el Museo Nacional de El Salvador. Museo nacional de Antropología, San Salvador, 2003.

Batres Posada, J.O. Descripción de los museos nacionales en El Salvador. Museo Nacional de Antropología, San Salvador, 2013.

Batres Posada, J.O. Los museos regionales. Museo Nacional de Antropología, San Salvador, 2013.

Batres Posada, J.O. Semblanza del museo nacional de El Salvador. Museo Nacional de Antropología, San Salvador, 2013. 
Cerna Chavarría, E. y Velásquez, J. Materiales para el estudio de David J. Guzmán. Datos biográficos concretos referentes al Dr. David Joaquín Guzmán, 1845-1945. Proyectos de investigaciones Antropológicas, Geográficas e Históricas. 1979.

Francia, Educación, Cultura, Religión, Lenguas, Música, Biblioteca y Museos. Disponible en: https://publicaronline.wordpress.com/2008/10/15/francia-educacion-cultura-religionlenguas-musica-biblioteca-y-museos/

Kuny Mena, E. Documento Único de la Biblioteca Especializada del Museo Nacional de Antropología, Historia de Patrimonio Cultural, San Salvador, 1983.

Linares Pérez, J.C. El museo, la museología y la fuente de información museística. Acimed 2008; 17 (4). Disponible en: http://eprints.rclis.org/12784/1/aci05408.pdf

Museo Nacional de Antropología, Decreto de Creación. Disponible en: http://mi.cultura.gob. sv/index.php/informacion/dependencias/museos/81-museo-nacional-de-antropologia-drdavid-j-guzman

Panameño, L. Historia de la Biblioteca Especializada. Biblioteca Especializada del Museo Nacional de Antropología, San Salvador, 2005.

Rubio Yánez, J. Antecedentes de la Creación de las Colecciones Nacionales de Bienes Culturales de El Salvador. Unidad de Control de Colecciones Nacionales. DRB. San Salvador, 2013.

\section{Entrevistas personales}

Sr. Jorge Rubio Yánez. Encargado de Depósitos de Colecciones de la Dirección de Registros e Inventarios. 2013.

Sra. Leticia Escobar de Vásquez, Centro de capacitación para la restauración, conservación y la promoción del patrimonio cultural en El Salvador. 2013.

Sr. Saúl Cerritos Peña, director del Museo Regional de Oriente en El Salvador. 2013. 


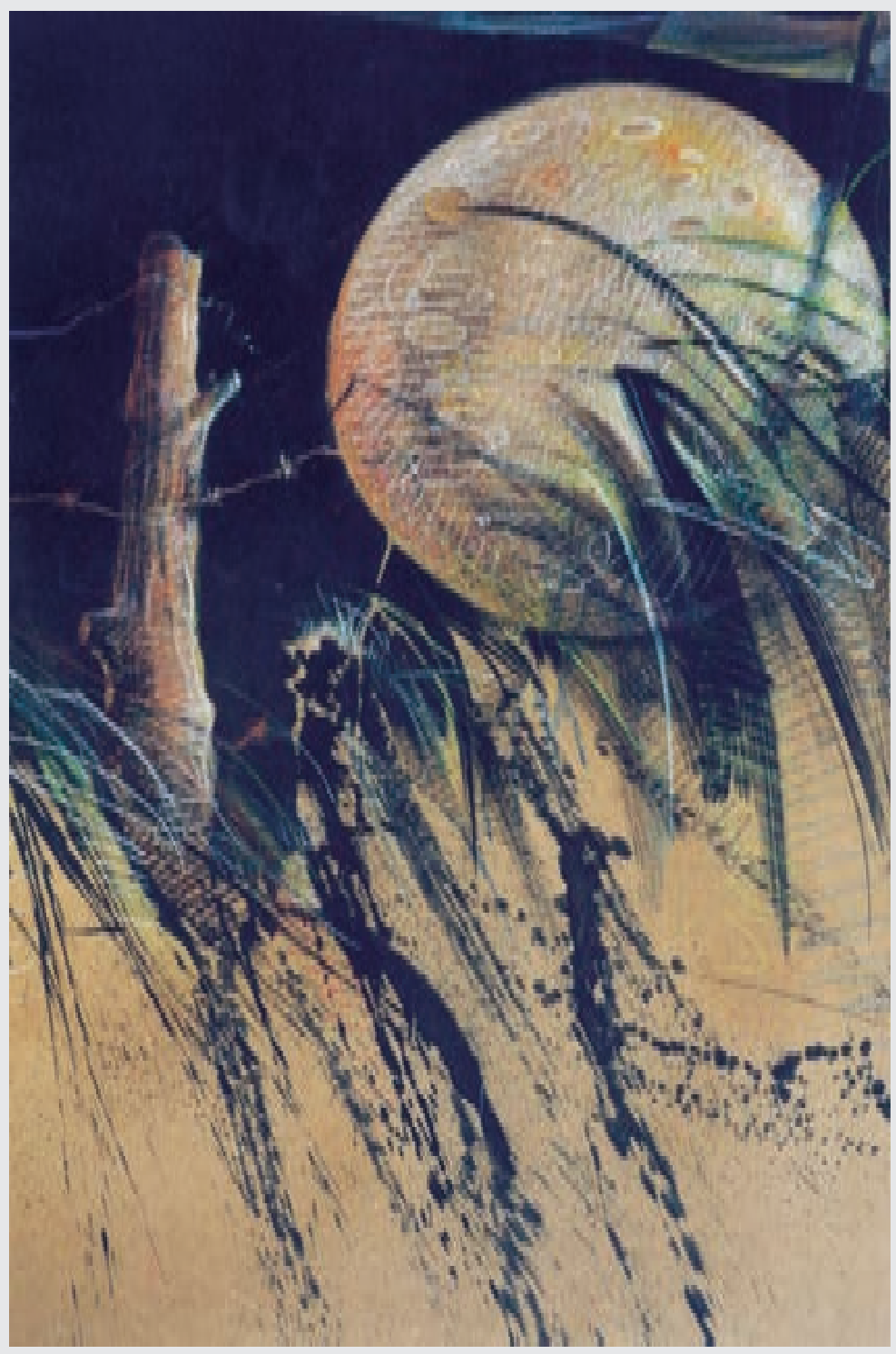

